\title{
A Novel Approach for Disaster Monitoring: Fractal Models and Tools
}

\author{
Gerardo Di Martino, Student Member, IEEE, Antonio Iodice, Senior Member, IEEE, \\ Daniele Riccio, Senior Member, IEEE, and Giuseppe Ruello, Member, IEEE
}

\begin{abstract}
In this paper, we present a complete framework to support the monitoring of natural and man-made disasters by means of synthetic aperture radar (SAR) images. The fractal geometry is the most appropriate mathematical instrument in describing the irregularity of a natural observed scene, by means of few effective and reliable parameters. Therefore, fractal concepts can be used to model and identify geometrical changes that occurred in areas hit by disasters. We present an overall framework employing fractal-based models, algorithms, and tools to support the identification of natural area changes due to natural or man-made disasters. Such a framework includes an algorithm used to extract fractal parameters from a 2-D signal, a fractal interpolation tool, and a SAR raw-signal simulator. The combined use of these tools provides an innovative instrument for disaster monitoring applications. In this paper, we implement the fractal framework to obtain a relation between the fractal parameters of a SAR image and those of the relative imaged area. In addition, a case study is discussed, showing the potentiality of our framework for flooding detection.
\end{abstract}

Index Terms-Change detection, fractals, synthetic aperture radar (SAR).

\section{INTRODUCTION}

$\mathbf{H}$ UMAN BEINGS live in an environment in continuous evolution, with a large number of physical phenomena which are potentially dangerous for their lives. Remote-sensing tools provide a great amount of data to be used in disaster prevention, risk evaluation, damage estimation and aid organization. However, practical use of these data is often limited by the lack of efficient, possibly unsupervised, tools for the retrieving of effective information to be employed in the crisis and postcrisis phases.

Several approaches devoted to define instruments and tools for data interpretation were presented in literature, showing, at least in principle, the potentiality of satellite and aerial technique for the monitoring and eventually prevention of natural (flooding [1], [2], volcanic risk [3], landslides [4], etc.) and human-made disasters (oil spills [5], fires [6], etc.). Most of these approaches are based on empirical analyses of remotesensing data, essentially driven by user needs. These analyses

Manuscript received May 1, 2006; revised September 29, 2006

G. Di Martino and G. Ruello are with Dipartimento di Ingegneria Elettronica e delle Telecomunicazioni, Università di Napoli "Federico II," 80125 Napoli, Italy and also with Ingegneria Senza Frontiere Napoli, 80128 Napoli, Italy (e-mail: gerardo.dimartino@unina.it; ruello@unina.it).

A. Iodice and D. Riccio are with the Dipartimento di Ingegneria Elettronica e delle Telecomunicazioni, Università di Napoli "Federico II," 80125 Napoli, Italy (e-mail: iodice@unina.it; daniele.riccio@unina.it).

Digital Object Identifier 10.1109/TGRS.2006.887024 are generally supervised, and to be effective, it is often required that the supervisor holds a remarkable level of competence with reference both to the remote sensors (and data) and to the effects of different disasters on the environment.

Among the remote sensors, the imaging ones take the advantage of generating synoptic views of the area under observation; in this case, the rationale for the feature identification techniques is generally based on the concepts of image texture analysis. Textures on remotely sensed images are related to morphological and geological features, land use, and social organization of the observed scene. An expert user can identify significant classes of human signatures, as ordered patterns which are well described within the classical geometry, and distinguish them from natural features that conversely hold selfsimilar characteristics, thus being governed by fractal laws [7]. When a disaster occurs, the scenario of the observed scene dramatically changes, and remote-sensing instruments should be, at least in principle, able to detect the changes in the scenes. As a matter of fact, man-made structures can be damaged thus (partially) loosing their classical-geometry properties; alternatively, some natural features can be modified, thus changing the characteristics of their fractal statistics. An example for the first happening is provided by images of urban areas stricken by earthquakes, where some chaotic textures appear and classical geometrical patterns are mixed with self-similar fractal ones. Examples for the second happening are provided by images of a flooding in rural areas or a volcano eruption; these natural disasters modify (according to different rules) the surface profile from scales smaller than the sensor coverage, but comparable to the sensor resolution, up to scales comparable to the electromagnetic wavelength.

Then, a fundamental aid in managing postcrisis analysis can be given by unsupervised or semiunsupervised tools for the interpretation of geometrical features in remotely sensed images: to develop these tools, it is crucial to introduce appropriate models to understand and quantitatively describe the physical phenomena that govern the modification of the scenario textures, thus providing a fundamental background to plan any powerful instruments to retrieve the information of interest.

As far as the models are under concern, the fractal geometry has the required characteristics in managing the problem at hand, because it simply accounts for geometrical irregularity of the observed objects. Therefore, fractal-based instruments are appropriate candidates for the retrieval of the significant physical parameters from remotely sensed images.

We focus our attention on synthetic aperture radar (SAR) sensors, because they are particularly effective in monitoring 
disaster events. From one side, the all-weather all-time characteristics of microwave images guarantees the monitoring of the scene where the disaster occurred; for instance, monitoring via optical images is dramatically reduced by the presence of clouds during flooding or of dust and smoke during volcano eruption. From the other side, in comparison with optical instruments, the microwave frequencies employed by radar instruments and the obtained geometric resolutions are better tailored to exploit the geometrical features of the area under survey: as already stated, these features exhibit the major changes whenever disasters take place.

In this paper, we present a novel fractal framework, based on direct and inverse models, to facilitate the disaster monitoring from SAR images. In particular, we propose the combined use of an appropriate SAR raw-signal simulator with fractalbased models and tools, according to the rationale described in Section II.

As for the direct chain, fractal geometrical models are employed to correctly represent the imaged surfaces [7]-[10], and fractal scattering models are employed to evaluate the reflectivity function of the natural scene under observation [11]. The evaluation of the scattering requires the knowledge of the fractal parameters of the area. Some techniques have been reported in the open literature for the retrieval of the fractal parameters from a given 2-D data set, most of them relying on the fractional Brownian model (fBm) [12]. In Section III, the $\mathrm{fBm}$ is described together with the main concepts which allow the development of the fractal inverse geometrical tool (IGT) and the fractal interpolation tool (FIT), whose details are discussed in [21].

The reflectivity function is employed to simulate the SAR raw signal and the relative SAR image, as described in Section IV. Simulated SAR images provide an extremely useful support for the interpretation of actual SAR signal formation mechanisms as well as for testing retrieving techniques.

As for the inverse chain, in the open literature, most of the change detection algorithms are based on ratioing and differencing of the magnitude of the signal return between pre and postcrisis scenario [14], [15]. Fractal tools of Section III could be employed in retrieving SAR properties of the SAR images and in developing change detection algorithms based on differencing and ratioing between the fractal parameters. Anyway, these techniques have been mainly proposed with reference to fractal surfaces; thus, they quite conveniently apply to digital elevation model (DEM); conversely, their use, in case of images of the fractal surface, is sometimes questionable. However, these methods present a major problem for remote-sensing applications: the estimation is effective only in the presence of data equally sampled in both directions. In Section V, a solution for the problem is proposed.

Such an approach calls for an appropriate discussion on the SAR image fractal parameter dependence on the observed scenario fractal parameter. No analytical solution was found so far to the problem. Anyway, the availability of direct and inverse models allow their combined use in creating a set of experiments to get an empirical relation between the fractal properties of the observed scene and those of the corresponding SAR image. The study is presented in Section VI.
Section VII is devoted in placing our models in the context of the actual data. A comparison between the SAR raw-signal simulator and actual images allows the validation of the whole direct chain. In addition, the direct chain is used to create canonical disasters in order to develop ad hoc solutions for a flooding test-bed. In Section VIII, we draw the final conclusions.

\section{FRACTAL FRAMEWORK}

The rationale for the overall fractal framework is presented in this section. The framework makes use of some tools which are here described in terms of input and output data, as well as of employed models and algorithms. The employed models and algorithms are discussed in detail in the following sections.

Monitoring of each type of possible disaster calls for an appropriate remote-sensor coverage, temporal and spatial resolution scales, and sensitivity.

Sensor coverage and sensitivity are somehow dual concepts. Sensor coverage allows focusing on the entire area involved in the disaster, so that large-scale phenomena can be monitored. Sensor sensitivity is here referred to the employed electromagnetic wavelength and can be related to the scene spatial scales that mainly affect the remotely sensed data, thus providing information on the small-scale phenomena. Temporal resolution fixes the time for obtaining postcrisis data and the average temporal lag between pre and postcrisis data. Spatial resolution allows the monitoring of the disaster at a significant scale with respect to the observed feature. This scale is set at an intermediate level between the sensor coverage and electromagnetic wavelength.

With respect to the aforementioned parameters, spaceborne and airborne SAR data provide a unique tool in monitoring several types of disasters. SAR coverage allows imaging earth at a regional scale, which is typical of most of possible disasters. SAR sensitivity depends on the backscattering properties at microwaves, thus being related to the geometrical properties of the scene under survey at centimetric and metric scales. The acquired SAR data exhibit a spatial resolution that can be employed to monitor the geometrical properties at scales ranging from $1 \mathrm{~m}$ to $1 \mathrm{~km}$. Finally, the new generation of spaceborne sensors is conceived to reduce the temporal resolution from days to hours, just as required for an efficient monitoring (and support controlling) of major natural and human-made disasters. Then, SAR images, with respect to optical ones, exhibit an emphasized dependence on the observed geometrical features on spatial scales ranging from several kilometers to some centimeters.

This short and general discussion provides the major motivation to propose a fractal framework for the study of disasters by means of SAR images. Fractal geometry provides the appropriate "environment" in dealing with geometric features that extend in such a wide range of scales. The fractal framework we propose is then based on the following tools.

1) A fractal IGT to retrieve the fractal parameters from the surface profile of the scene under analysis: Input of this tool is an (original) DEM of the scene under analysis. This DEM can be acquired by aereophotogrammetric campaigns or interferometric SAR data; it should be 
relevant to the entire area under analysis and should be sampled with a spacing as close as possible to the SAR resolution. In general, a coarse version of these DEMs can be provided by the data from the Shuttle Radar Topography Mission. These outputs of this tool are the maps containing the fractal parameters evaluated in each point of the original DEM of the scene. The tool we propose is based on the $\mathrm{fBm}$ for the DEM, whose details are provided in Section III. The employed algorithm is based on the variogram analysis and is detailed in Section V.

2) A FIT to obtain the surface profile sampled according to the resolutions of the considered SAR sensor and stochastically described at the scales comparable to the employed wavelength: The input for this tool are the original DEM and the maps of the fractal parameters evaluated by the tool described at point 1). Output is a realization of the macroscopic DEM at the SAR resolution scale. At the microscopic scale, only the fractal parameters are required by the scattering models, and the $\mathrm{fBm}$ model is employed to fix these fractal parameters equal to those estimated at the original scale, thus performing a stochastic interpolation of the scene profile up to the scale typical of the electromagnetic wavelength.

3) A SAR simulator (SARAS) that makes use of fractal direct scattering formula to generate raw signals and images relevant to the scene under analysis: Main inputs of this tool are the realization of the fractal profile of the scene sampled at the SAR resolution scale and the fractal parameters at the SAR wavelength scale. This tool evaluates for each portion of the scene the backscattering via a direct scattering formula and projects this result onto the azimuth-slant range coordinate systems. This tool employs an $\mathrm{fBm}$ model for the surface (see Section III); algorithms for the SAR sensor are based on what is reported by the study in [13] and summarized in Section IV. The tool can alternatively employ physical optics (PO) or small perturbation method (SPM) fractalbased solutions for scattering, whose fundamentals are reported under Section IV.

4) A fractal inverse tool (ITR) to retrieve the fractal parameters from the simulated images: Inputs of this tool are the simulated SAR images of the area. The outputs of this tool are the maps containing the fractal parameters evaluated in each point of the simulated images. The tool we propose is based on the $\mathrm{fBm}$ for the simulated images (see Section III). The employed algorithm can be based on the variogram analysis and is detailed in Section V. An assessment of this tool is required: this is given in Section VI, thus showing the algorithm performances whenever SAR images are considered and some disasters occur.

\section{Fractal Models For IGT AND FIT}

Fractal models are widely considered the most appropriate to quantitatively describe natural surfaces [7]-[10]. Fractal geometry is able to simply account for the nonstationarity of natural surfaces, as well as for their self-affinity. The most used fractal model is the fBm [16], [17], whose realizations can be also obtained by employing the Weierstrass-Mandelbrot (WM) function, which is a continuous but not differentiable function [18]-[20]. The $\mathrm{fBm}$ is defined in terms of the probability density function of its height increments: a stochastic process $z(x, y)$ is an fBm surface if, for every $x, y, x^{\prime}, y^{\prime}$, it satisfies the following relation:

$\operatorname{Pr}\left\{z(x, y)-z\left(x^{\prime}, y^{\prime}\right)<\bar{\zeta}\right\}=\frac{1}{\sqrt{2 \pi} s \tau^{H}} \int_{-\infty}^{\bar{\zeta}} \exp \left(-\frac{\zeta^{2}}{2 s^{2} \tau^{2 H}}\right) d \zeta$

where $\tau$ is the distance between the points $(x, y)$ and $\left(x^{\prime}, y^{\prime}\right)$, and the two parameters that control the $\mathrm{fBm}$ behavior are as follows:

- $H$ : the Hurst coefficient $(0<H<1)$, related to the fractal dimension $D$ by means of the relation $D=3-H$;

- $s$ : the standard deviation, measured in $\left[m^{(1-H)}\right]$, of surface increments at unitary distance, a real parameter related to an $\mathrm{fBm}$ characteristic length, the topothesy $T$, by means of the relation $s=T^{(1-H)}$.

For a given surface, the structure function (variogram) $V(\tau)$ is defined as the mean-square increment of elevation points placed at distance $\tau$

$$
V(\tau)=\left\langle\left(z(x, y)-z\left(x^{\prime}, y^{\prime}\right)\right)^{2}\right\rangle .
$$

The variogram of an $\mathrm{fBm}$ surface can be evaluated in terms of the parameters $H$ and $s$ as

$$
V(\tau)=s^{2} \tau^{2 H} .
$$

Equation (3.3) can be written in logarithmic form as

$$
\log V(\tau)=2 \log s+2 H \log \tau
$$

which defines in a $\log -\log$ plane a linear behavior with slope $2 H$ and ordinate intercept $2 \log s$.

It has been demonstrated [7]-[9] that the spectrum $S(k)$ of an isotropic $\mathrm{fBm}$ process exhibits a power law behavior

$$
S(k)=S_{o} k^{-\alpha}
$$

wherein the spectral and spatial domain parameters are related [11] by the following relationships:

$$
\begin{gathered}
\alpha=2+2 H=8-2 D \\
S_{0}=s^{2} 2^{2 H} 2 \pi H \frac{\Gamma(1+H)}{\Gamma(1-H)}
\end{gathered}
$$

with $\Gamma(\cdot)$ being the Gamma function. From the inequalities $0<$ $H<1$, we get $2<\alpha<4$, which defines the range of allowed values for the spectral slope $\alpha$. Note that also the spectral law (3.5) provides a linear relation in a $\log (S)-\log (k)$ plane, with parameters related to those of the $\log -\log$ representation introduced for the variogram.

Based on the $\mathrm{fBm}$ model, the IGT can be developed by exploiting the linear dependence in the $\log -\log$ plane of the spectrum and the variogram, recovering the $H$ and $s$ values 


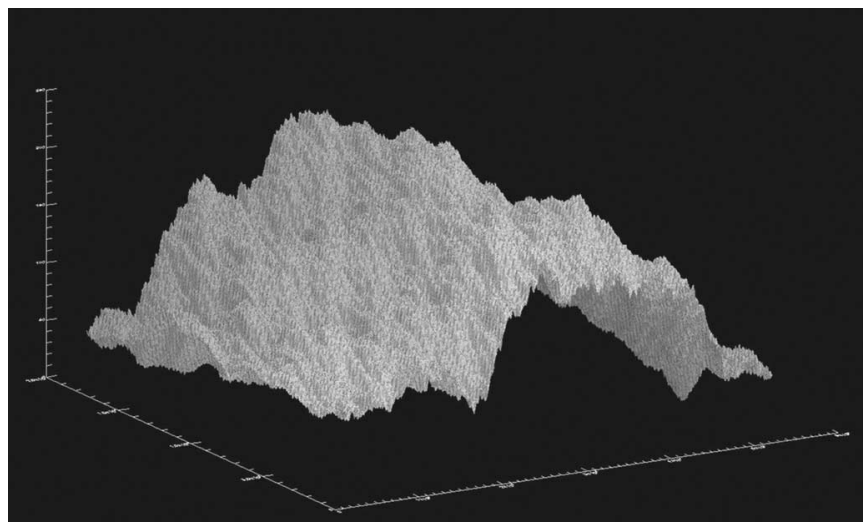

Fig. 1. Canonical fractal surface with fractal dimension $D=2.3$ and $s=$ $0.1 \mathrm{~m}^{0.3}$.

TABLE I

$s$ AND $H$ MEAN VALUES AS A FUNCTION OF THE Window DimENSION

\begin{tabular}{|c|c|c|c|c|}
\hline \hline $\mathrm{W}$ & $\mathrm{E}[D]$ & $\operatorname{stdev}[D]$ & $\mathrm{E}[s]$ & $\operatorname{stdev}[s]$ \\
\hline 5 & 2.367 & 0.177 & 0.107 & 0.029 \\
\hline 9 & 2.349 & 0.079 & 0.102 & 0.011 \\
\hline 11 & 2.348 & 0.0643 & 0.102 & 0.0078 \\
\hline
\end{tabular}

by performing a linear regression over the log-log plots of measured values of $V(\tau)$ or $S(k)$ [11]. In addition, the FIT can be developed exploiting the fBm definition, as discussed in detail in [21].

\section{SARAS}

The interpretation of SAR data is often difficult, due to the fact that SAR images are related by nonlinear relations with the scene parameters. Therefore, it is crucial to have efficient tools able to predict the SAR data behavior as a function of the scene parameters. A SAR raw-signal simulator was developed and tested [13], [21]; its use provides an added value information for SAR data interpretation and a support for SAR processing techniques (for instance, image classification, identification of flooded areas, and so on).

Let $x$ and $r$ be the independent space variables, standing, respectively, for azimuth and range. By using primed coordinates for the independent variables of the SAR raw signal $s\left(x^{\prime}, r^{\prime}\right)$, this can be expressed as [13]

$$
s\left(x^{\prime}, r^{\prime}\right)=\iint d x d r \gamma(x, r) g\left(x^{\prime}-x, r^{\prime}-r ; r\right)
$$

where $\gamma(x, r)$ is the reflectivity pattern of the scene and $g\left(x^{\prime}-\right.$ $\left.x, r^{\prime}-r ; r\right)$ is the unit impulse response of the SAR system [13], [21]. Evaluation of the reflectivity pattern requires a description of the observed surface as well as a model for their interaction with the electromagnetic fields radiated by the SAR antenna [13].

The presented simulator was developed by assuming that the observed surfaces could be modeled as pure Gaussian functions. Theoretical [7]-[9] and experimental [22] studies suggest that the use of fractal models improve the scattering method results. In this paper, we used the $\mathrm{fBm}$ fractal model for describing the surface roughness and the SPM as scattering

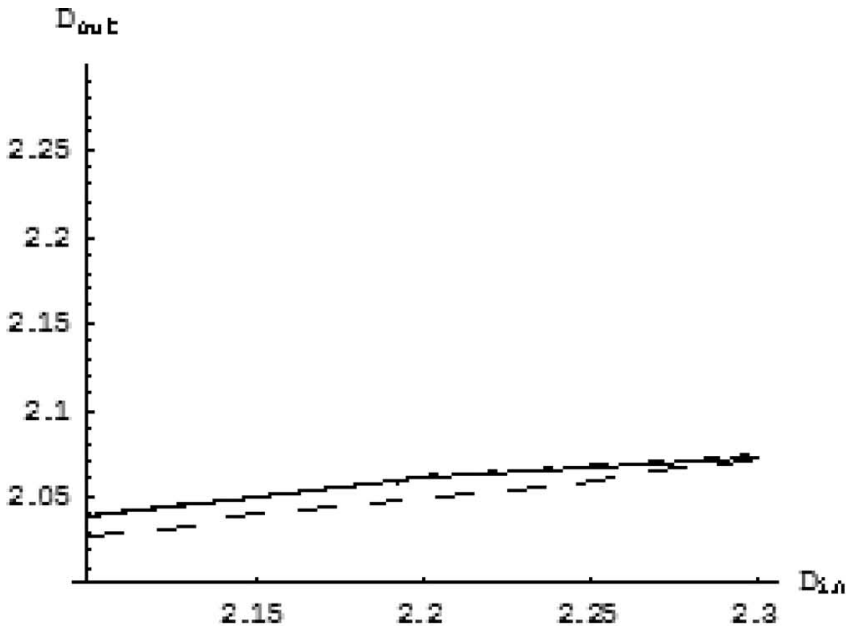

(a)

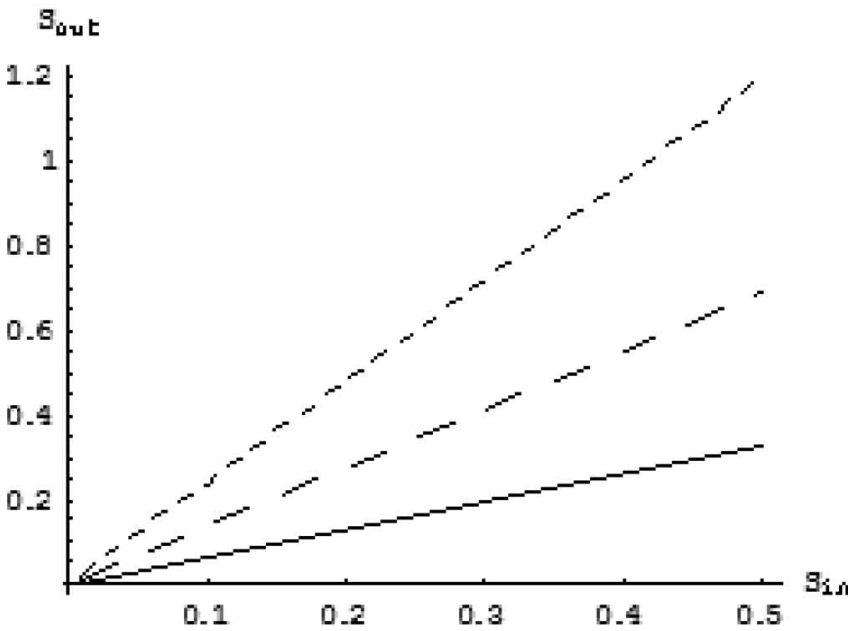

(b)

Fig. 2. (a) Fractal dimension of the SAR image as a function of the fractal dimension of the scene for $s=0.01 \mathrm{~m}^{0.3}$ (continuous line), $s=0.1 \mathrm{~m}^{0.3}$ (long dashed line), and $s=0.5 \mathrm{~m}^{0.3}$ (short dashed line)-the short dashed and the continuous lines are overlapped. (b) $s$ parameter of the SAR image as a function of the $s$ parameter of the scene for $H=0.9$ (continuous line), $H=0.8$ (long dashed line), and $H=0.7$ (short dashed line).

model for evaluating the reflectivity pattern. Accordingly, the radar cross section $\sigma_{\mathrm{pq}}^{0}$ required for the $\gamma(x, r)$ evaluation can be written as

$$
\sigma_{\mathrm{pq}}^{0}=8 k^{4} \cos ^{4} \vartheta\left|\beta_{\mathrm{pq}}\right|^{2} 2 \pi \frac{S_{0}}{(2 k \sin \vartheta)^{\alpha}}
$$

where $\beta_{\mathrm{pq}}$ is a function accounting for the field polarization [11], $\vartheta$ is the local incident angle, and $k$ is the electromagnetic wavenumber.

Note that (4.2) diverges at a normal incidence. Such a condition does not allow the implementation of the model in practical cases. Therefore, in order to overcome this problem, we make use of a transition function for low incidence angles, based on the PO solution to the Kirchhoff approach for $\mathrm{fBm}$ surfaces [16].

Comparison between simulated and actual SAR data was presented in [21], with respect to image single point normalized 


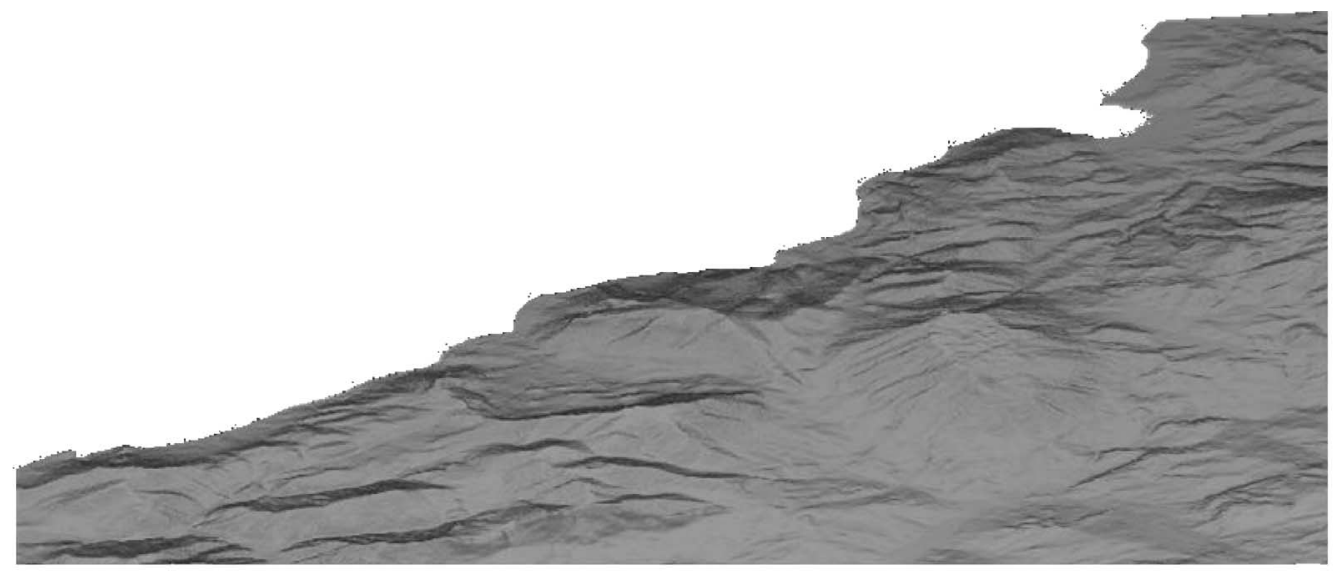

(a)

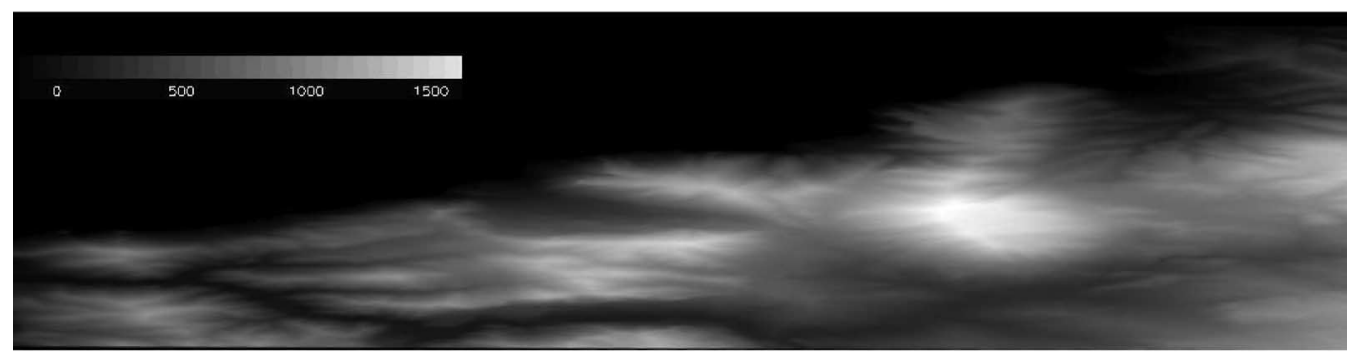

(b)

Fig. 3. (a) Three-dimensional representation of the Maratea area, as seen by the ERS-1 SAR sensor (near range is on the bottom). (b) Grayscale representation of the interpolated $17.464 \times 56.641 \mathrm{~km}^{2} \mathrm{DEM}$ of the Maratea area, with $3.99 \times 19.93 \mathrm{~m}^{2}$ pixel spacing.

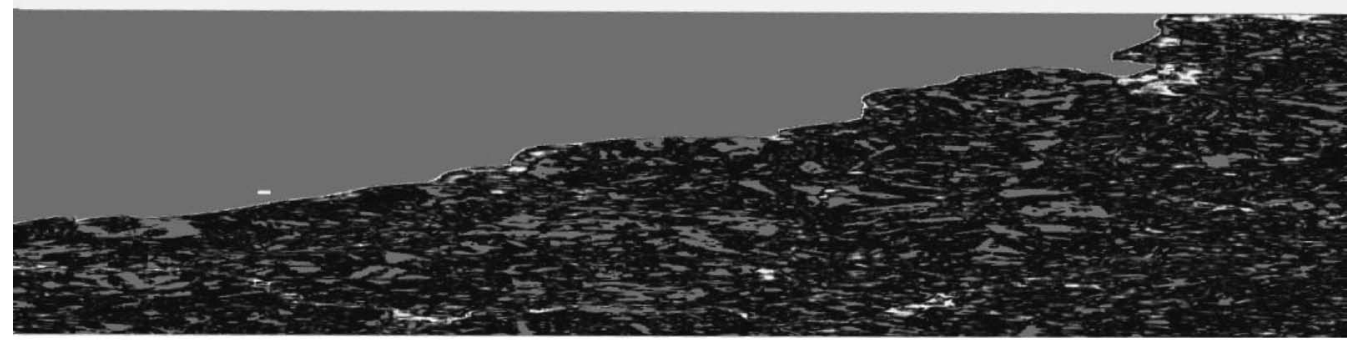

(a)

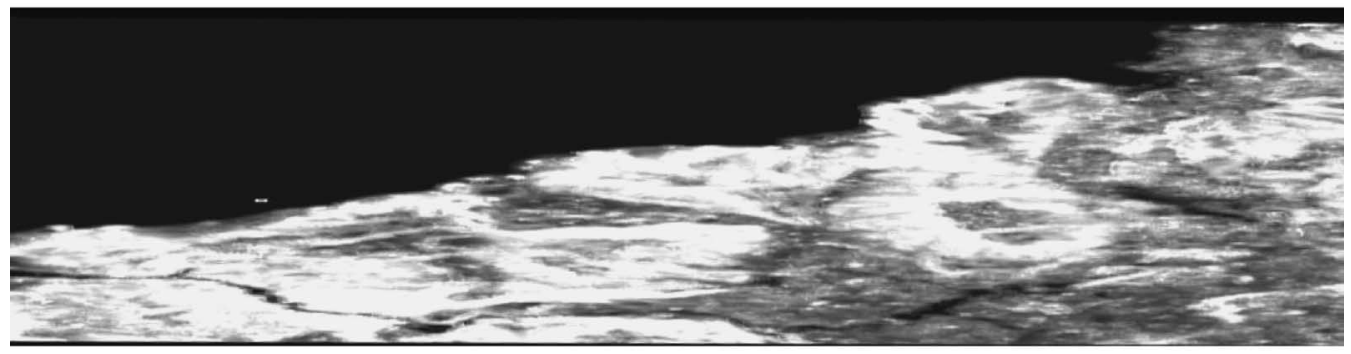

(b)

Fig. 4. (a) Fractal dimension and (b) $s$ parameter maps relative to the interpolated $17.464 \times 56.641 \mathrm{~km}^{2}$ DEM of the Maratea area, with $3.99 \times 19.93 \mathrm{~m}^{2}$ pixel spacing.

moments and autocorrelation function, thus assessing the simulator reliability. In those comparisons, the fractal parameters employed for the macroscopic DEM were assumed to be variable at the macroscopic scale (SAR resolution). In this case, the extension we propose allows considering also for the microscopic scale (up to the electromagnetic wavelength scale) the fractal parameters estimated from the available DEM, so that, in order to compute the reflectivity function, we use fractal parameters varying over the scene.

\section{RETRIEVING OF THE FRACTAL PARAMETERS (ITR)}

A disaster brings significant changes in the geometrical properties of the affected scene. As an example, the roughness 


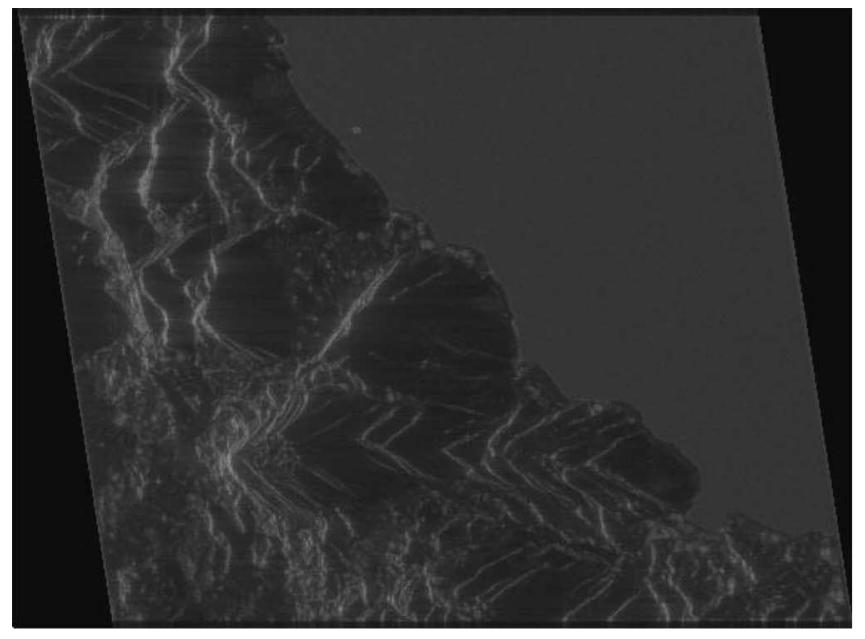

(a)

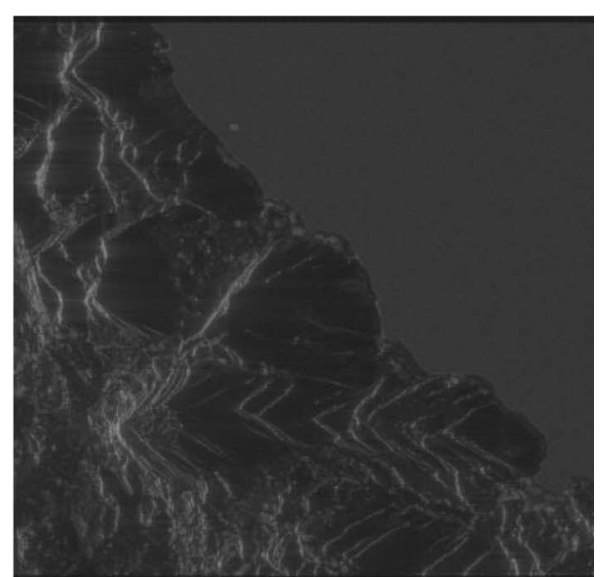

(b)

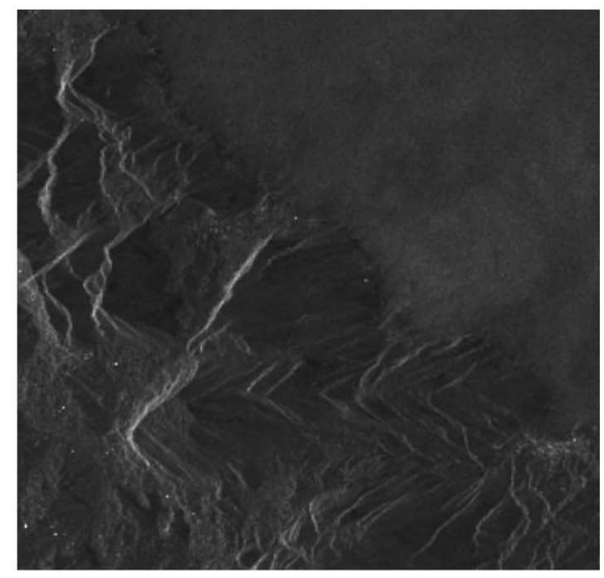

(c)

Fig. 5. (a) Simulated $2 \times 10$ multilook ERS-1 C-band SAR image relative to the area of Maratea (near range is on the left) with $39.86 \times 15.81 \mathrm{~m}^{2}$ azimuth-slant range resolution, corresponding to a $39.86 \times 37.58 \mathrm{~m}^{2}$ azimuth-ground range resolution. A comparison between (b) simulated and (c) actual ERS-1 SAR image (only in the area where both data are available).

of a flooded area is usually lower than before the event, while an inhabited area affected by an earthquake will lose the ordered organization typical of built-up quarters. As presented in Section III, fractal geometry accounts for the irregularity of the surfaces; therefore, its use is widely suggested for change monitoring [7]-[10].

In the following, we present a new technique for extracting fractal parameters of an observed scene and a possible algorithm for identifying zones affected by a disaster.

For a 2-D fBm process $f(x, y)$, it is possible to write the mean value of the increments over a fixed distance $\tau$ as

$$
E[|f(x+\Delta x, y+\Delta y)-f(x, y)|] \cdot \tau^{-H}=C
$$

where the constant $C$ can be obtained from (3.1)

$$
C=\frac{2}{\sqrt{2 \pi}} s .
$$

Equation (5.1) is equivalent to

$\log E[|f(x+\Delta x y+\Delta y)-f(x, y)|]-H \log \tau=\log C$.
Therefore, the plot of $E[|f(x+\Delta x, y+\Delta y)-f(x, y)|]$ as a function of $\tau$ on a $\log -\log$ plane lies on a straight line of slope $H$ and whose intercept with the $\log E[\mid f(x+\Delta x, y+\Delta y)-$ $f(x, y) \mid]$ axis is $\log C$. Our algorithm retrieves the $H$ and $s$ values by fitting a straight line to the computed fractal data.

In the case of $2-\mathrm{D} \mathrm{fBm}$ processes, such a procedure can be used in retrieving the fractal parameters, provided that the considered surface is isotropic. In this case, we can use a sliding window, whose movements cover the whole surface, and we can evaluate the absolute values of the increments over all the possible couple of points with the same distances. Such an approach is applicable if, in the considered surface, there is no preference directionality.

In this paper, we extended the Yokoya's method [12] to deal with not equally spaced data. Note that, if the data are nonequally spaced, the number of points with the same relative distances is smaller than the equally spaced case, causing a decreased reliability of the statistics. In order to reduce this effect, we performed a linear regression using the points in the fractal plot, weighting them through an uncertainty function varying according to the number of increments over which we computed the expected value. 


\section{FrameWORK ASSESSMENT: StUdiES ON Fractal CANONICAL SURFaces}

A SAR image is the result of nonlinear phenomena involving the physical parameters of the observed scene: electromagnetic scattering, ground to slant range mapping, shadowing, etc. In the open literature, a closed-form expression for the relationship between SAR image and scene fractal parameters cannot be found. In order to shade light on the very involved dependence of the image characteristics on the scene parameters and to test the retrieval algorithm, we present, in this section, a quantitative analysis of the approach described in Section II for a canonical fractal surface, i.e., a synthesized fractal surface with fixed fractal parameters.

We synthesized the canonical fractal surface by means of the WM stochastic function [8], [18], [22]: the fractal parameters are fixed to $H=0.7$ and $s=0.1 \mathrm{~m}^{0.3}$, which are typical values for natural surfaces [23]. The surface shape is presented in Fig. 1. According to our framework, the obtained DEM is provided as an input of the SARAS once it is sampled at the scales of the resolution of the ERS-1 C-band SAR sensor we selected for the test.

The fractal parameters of the scene can be retrieved via the IGT of Section V. To implement that algorithm, it is preliminarily required to choose sliding window dimension for the fractal parameters retrieval procedure. Therefore, we tested the dependence of the algorithm results on square sliding windows whose dimensions ranged from 5 to 11 pixels. The obtained average $H$ and $s$ mean values are presented in Table I. As expected, the higher the window dimensions, the closer is the value of the estimated fractal parameters to the actual ones. Such an improvement of the results is paid in terms of computation complexity and, as a consequence, of processing time. In particular, the time elapsed for elaborating a $200 \times 200$ pixel image with a 1.9-GHz personal computer moves from $3 \mathrm{~s}$ if we employ a $3 \times 3$ pixel window, to $153 \mathrm{~s}$ with a $11 \times 11$ pixel window. A larger window can be employed if computational time is not a critical issue, and this is the case for a canonical surface. However, in actual cases, the fractal dimension can change across the considered area; in these cases, the larger the sliding window dimensions, the higher is the correlation for the fractal parameters estimated at adjacent points, thus the lower the spatial resolution of the fractal parameters estimation process. The latter implying that smaller fractal features can be lost. In the following, both for the canonical and actual fractal surfaces, we set the sliding window dimension to $9 \times 9$ pixels: this value represents a convenient tradeoff between precision and spatial resolution (and, obviously, processing time) for the actual cases considered in this paper.

Once the window dimension has been chosen, it arises the need of understanding how the fractal parameters are accounted for in the SAR images. In the following, as far as the microscopic scale is concerned, we present the dependence of the algorithm results on their values. Therefore, we used the sampled WM canonical fractal function as an input for the SARAS, in accordance with point 3) of the framework presented in Section II. The SAR image was then obtained via standard SAR raw data processing, and the fractal parameters of the

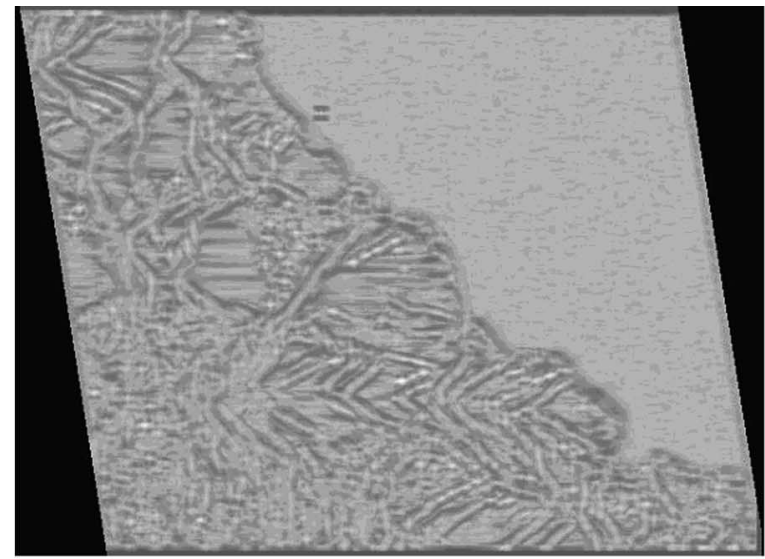

(a)

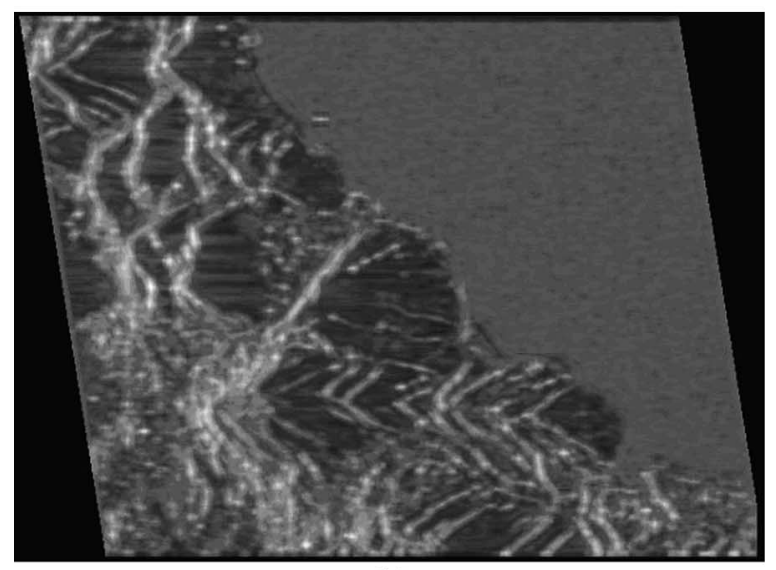

(b)

Fig. 6. (a) Fractal dimension and (b) $s$ parameter maps relative to the simulated SAR image of the precrisis scenario with $39.86 \times 15.81 \mathrm{~m}^{2}$ azimuthslant range resolution.

images are retrieved in accordance with the fractal inverse tool referred to at point 4) (Section II). To obtain multilook images, a spatial averaging of $2 \times 8$ pixels was employed. We repeated the SAR simulation for different values of the canonical surface fractal parameters. In Fig. 2, we plot the fractal parameters $D_{\text {out }}$ and $s_{\text {out }}$ values estimated on the SAR signal versus the input fractal parameters of the canonical profiles $D_{\text {in }}$ and $s_{\text {in }}$. In particular, in Fig. 2(a), it is shown that the fractal dimension of the SAR image does not significantly change as a function of the scene fractal dimension. Conversely, the $s_{\text {out }}$ values of the SAR image increase almost linearly with the parameters $s_{\text {in }}$ of the observed scene.

Such a result is particularly relevant because it allows obtaining an empirical relationship between SAR images and the scene fractal parameters, extremely useful for the interpretation of SAR images in a crisis scenario, as shown in the following section.

\section{Actual Case Studies}

The overall framework presented in the previous sections form a core, whose implementation can be tailored for several applications. In this section, we present some case studies with a twofold objective: a comparison of our techniques with actual 


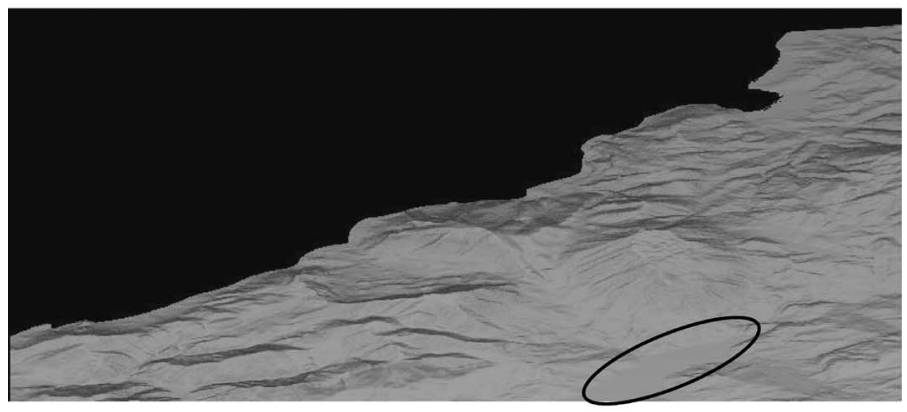

(a)

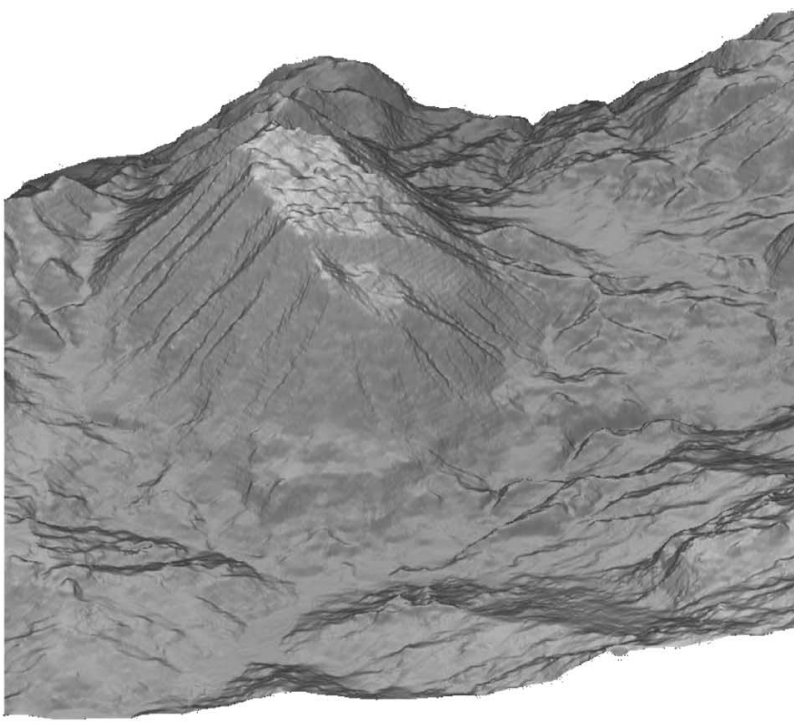

(b)

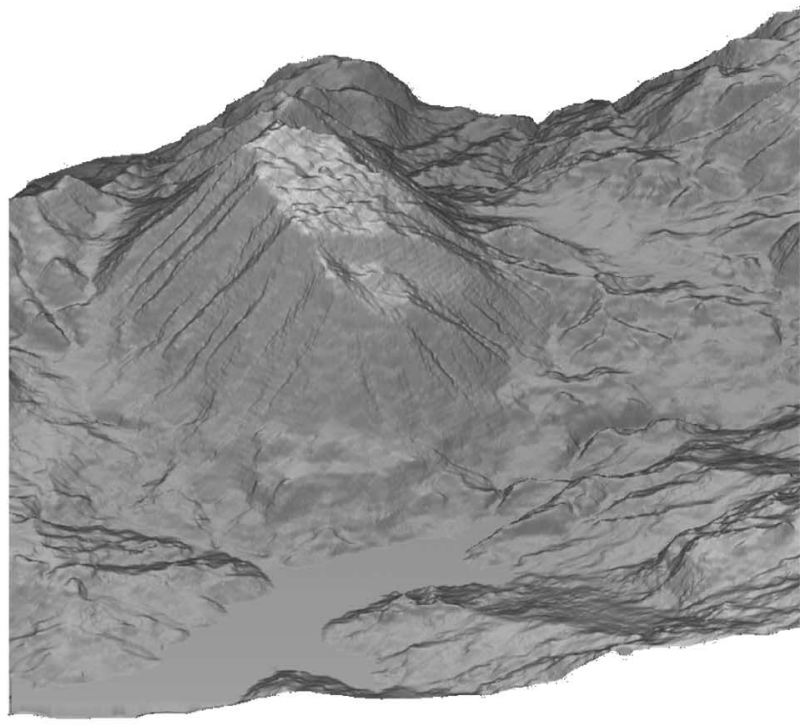

(c)

Fig. 7. (a) Three-dimensional representation of the Maratea region after the flooding, as seen by the ERS-1 SAR sensor (near range is on the bottom). Flooded area is marked in the black oval. A close up of the area hit by the flooding (b) before and (c) after the event.

cases and the demonstration of its potentiality for working in actual disaster monitoring.

\section{A. Comparison With Actual SAR Images}

The region of interest is the area of Maratea $\left(39^{\circ} 59^{\prime} \mathrm{N} 15^{\circ} 42^{\prime} \mathrm{E}\right)$, south of Italy, a coastal area surrounded by steep mountains. A DEM of a $20 \times 20 \mathrm{~km}^{2}$ area, with a $20 \times 20 \mathrm{~m}^{2}$ pixel spacing was available for the considered area, as well as an ERS-1 C-band SAR image, acquired in descending orbit on January 30, 1996, with a view angle of $24.88^{\circ}$.

First, we used our DEM as input for the SARAS simulator, in order to compare the simulated with the actual ERS-1 image. The DEM was interpolated via the FIT procedure on a $3.99 \times 19.93 \mathrm{~m}^{2}$ spaced grid, in accordance with the ERS-1 acquisition geometry. A pictorial view of the interpolated DEM is shown in Fig. 3(a), with an observation angle of $24.88^{\circ}$, so that it reproduces the scenario seen by an observer on the ERS- 1 satellite.

In Fig. 3(b), the DEM grayscale representation is provided. Note that the highest mountain (corresponding to the brightest area) has a top altitude of $1506.45 \mathrm{~m}$. Columns of the image represent equi-azimuth pixels.

In order to use the presented DEM as input for the SARAS, the description of the scales at the order of the incident wavelength ( $5 \mathrm{~cm}$ for ERS-2 sensor) is needed. The fractal parameters of a natural surface are independent of the scale at which we measure them [11]; therefore, we retrieved the microscopic fractal parameters from the DEM via the IGT. We present the fractal dimension $D$ and the $s$ parameter estimated by the reference DEM in Fig. 4(a) and (b), respectively. Note that the $s$ map allows an identification of topographical characteristics, like rivers or mountains. 
Once the long- and short-scale characterization is provided, it is possible to perform the SARAS simulation of the image generated by the ERS-1 sensor.

The image obtained from the simulated raw signal via standard processing is presented in Fig. 5(a). The image is averaged with a $2 \times 10$ multilook, so that its resolution in azimuth-slant range is $39.86 \times 15.81 \mathrm{~m}^{2}$, which corresponds to an azimuthground range approximately square pixel $\left(39.86 \times 37.58 \mathrm{~m}^{2}\right)$.

In Fig. 5(b) and (c), we present the simulated and the actual ERS-1 images, respectively. A portion of the image was cut in order to depict the region where both the data were available. A visual comparison shows that the simulator is able to reproduce the main characteristics of the SAR image, suggesting the use of the SARAS as support for SAR actual image interpretation. Note that the main differences between the two images are in the sea and in presence of inhabited regions. This is due to the fact that, in the presented simulation, we did not use an ocean surface nor a city model.

As presented in the point 4) of Section II, we can estimate the fractal parameters of the SAR image with a fractal inverse tool (ITF), obtaining the maps of the fractal dimension and of the $s$ parameter for the SAR image presented in Fig. 6(a) and (b), respectively. The presented results suggest the use of the SARAS for simulating canonical scenarios to be used as test-bed for the validation of change detection techniques. Therefore, we simulated a crisis scenario relative to a flooding of the area adjacent to the highest mountain of the region of interest. The previous simulation is considered as the precrisis scenario, and it is used as reference for fractal change detection approaches, as detailed below.

\section{B. Flooding}

In the following, we present the potentiality of our framework applied to the monitoring of flooding. We modified the original DEM by creating a river's spate in the valley pinpointed by the black oval in Fig. 7(a). The mean difference between the pre and postcrisis DEM in that area is about $30 \mathrm{~m}$. A closeup of the flooded region before and after the disaster is presented in Fig. 7(b) and (c), respectively.

In order to appropriately simulate the presence of water in the flooded region, we modified the microscopic roughness and the dielectric parameters as well. As far as the microscopic fractal parameters are concerned, in the areas affected by the flooding, we set $H$ to a typical value for the water surface $(H=0.75)$, and we set $s$ to one half of the value in the precrisis scenario. As for the dielectric characterization, the area affected by the flooding is assumed to have a dielectric constant of $20 \varepsilon_{0}$ and a conductivity of $1 \mathrm{~S} / \mathrm{m}$, which are typical values for extremely wet terrain; the surrounding area is assumed to have a dielectric constant of $4 \varepsilon_{0}$ and a conductivity of $10^{-3} \mathrm{~S} / \mathrm{m}$, which are typical of terrains with low water content [24].

The SARAS simulated image corresponding to the postcrisis scenario is presented in Fig. 8. A visual comparison with Fig. 5(a) shows that the user can visually recognize the area hit by the flooding. Anyway, for actual cases, a quantitative technique to identify the area involved in the phenomenon is required, and its implementation is discussed in the following.

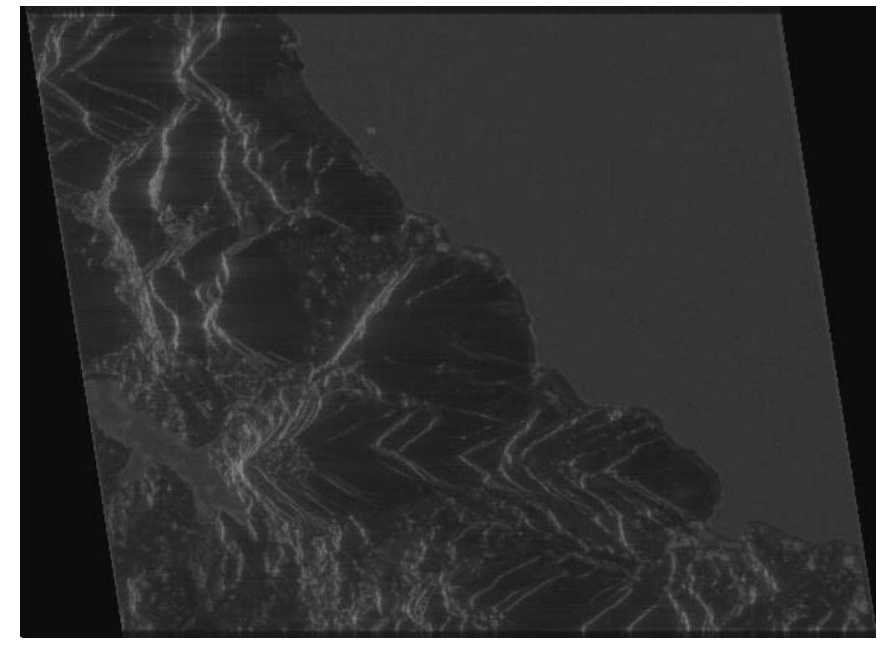

Fig. 8. Simulated $2 \times 10$ multilook ERS-1 C-band SAR image relative to the postflooding scenario (near range is on the left) with $39.86 \times 15.81 \mathrm{~m}^{2}$ azimuth-slant range resolution.

Therefore, the use of classical and fractal techniques is in order. A quantitative comparison is possible if a ground truth is available. Therefore, in order to define a reference map, we exploited the SARAS facilities of simulating the SAR image in the absence of speckle. Such an approach allows the definition of the "ground truth" in the SAR image by differencing pre and postcrisis intensity images in the absence of speckle and the creation of the reference mask of Fig. 9(a), where flooded regions are identified by white pixels.

Then, we tested the classical change detection technique based on the differencing between SAR intensity pre- and postcrisis images, obtaining a binary mask presented in Fig. 9(b). A visual comparison between Fig. 9(a) and (b) shows that the flooded area is well identified by the technique (we estimated that the hit rate is $97.2 \%$ ), but this is paid with an excessive false alarm rate (11.2\%) due to the fact that the multiplicative random noise due to the speckle in correspondence of layover areas can significantly change from a realization to another. Note that, due to the peculiar distribution of the misclassified pixels, it is not trivial to improve the technique performance via classical postprocessing algorithms.

Then, we implemented a change detection technique based on the fractal framework presented in the previous sections. In particular, we used the ITF to retrieve the fractal parameters of the pre and postcrisis scenario. In Fig. 9(c), we show a classification map obtained by the difference between the fractal dimensions of pre and postcrisis scenes. Again, it is possible to identify the flooded area with a good hit rate (83\%), but again, the false alarm rate is extremely high (15.4\%).

Anyway, by observing Fig. 9(b) and (c), we note that the distribution of misclassified pixels is completely different due to the different causes that generate it. In fact, the image intensity difference is very sensitive to the signal magnitude changes; therefore, most of the misclassified pixels are grouped in the layover areas (it is consistent with the fact that SAR signal decorrelation increases in layover areas). Conversely, the fractal dimension is more sensitive to gradients of the signal; therefore, most of the noise is gathered in correspondence 


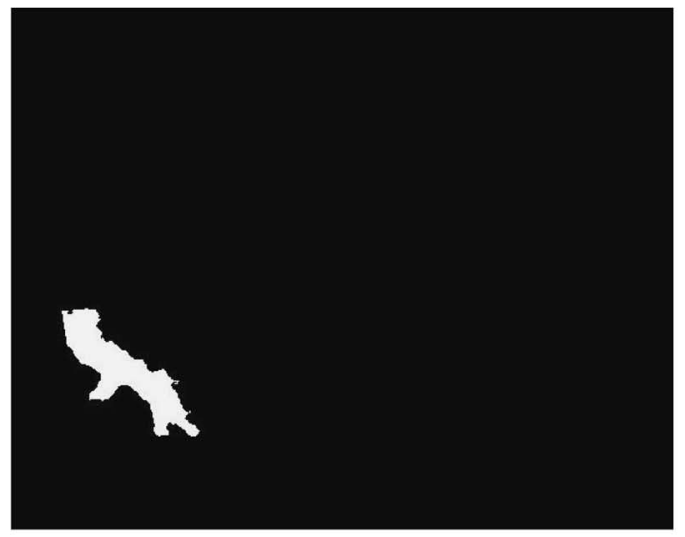

(a)

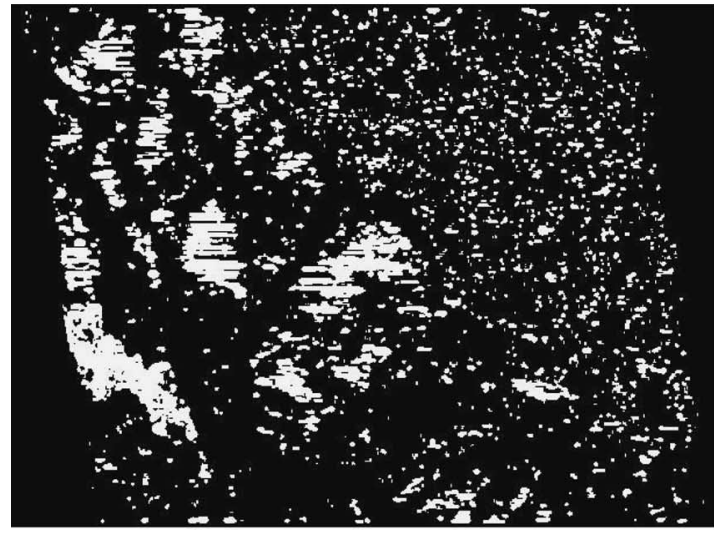

(c)

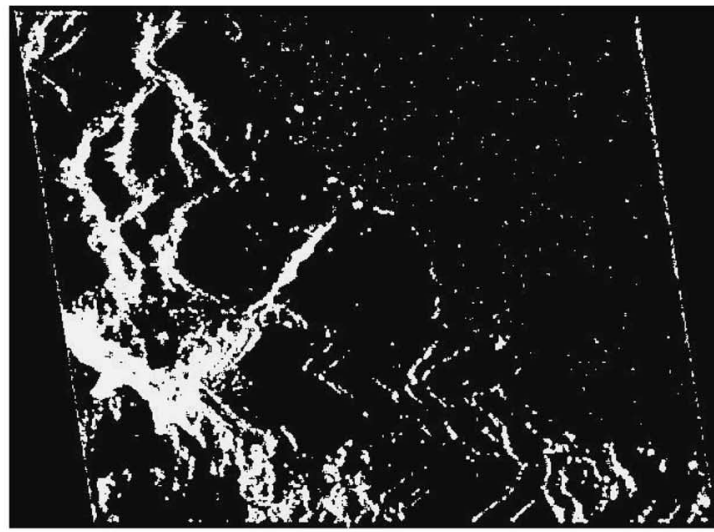

(b)

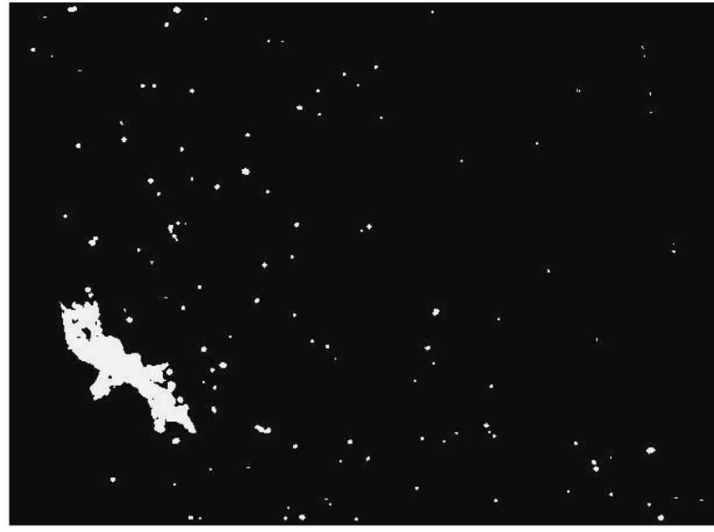

(d)

Fig. 9. (a) Reference classification map ("ground truth"). Classification maps obtained by (b) magnitude differencing, (c) fractal dimension differencing, and (d) combined technique.

of the grazing angle areas, where the differences between the side lobes of the layover areas create steep gradients. Above considerations suggest to combine the obtained results in order to get a significant improvement on the detection performances.

A simple multiplication of the obtained masks allows deleting most of the misclassified pixels, causing a strong reduction of the false alarm rate. Such a combined technique leads to a hit rate of $81.1 \%$, with a false alarm rate reduced to $0.5 \%$. A further low-complexity processing can consist in a smoothing devoted to throw out isolated misclassified pixels, obtaining an improvement of the hit rate to $90.2 \%$, paid with a false alarm rate raised to $0.6 \%$, corresponding to the classification mask presented in Fig. 9(d). Fig. 9(d) suggests that the residual false alarm error can be further reduced via simple postprocessing algorithms.

Note that, by changing the thresholds used for the classification maps, the results can slightly change. Anyway, the choice of the thresholds is beyond the scope of this paper; it can be done in accordance with the specific application, and it does not change the essence of above-presented results.

In addition, note that the $s$ information was not used because, in this peculiar case, it does not bring a significant classification improvement. This is mainly due to the fact that, due to the particular topography, the $s$ data and the image intensity information are highly correlated.

\section{CONCLUSION}

Fractal geometry proved to be a powerful instrument in representing natural surfaces, and its use in all the related applications is quickly increasing. Earthquakes, floodings, and volcanic eruptions change the geometrical properties of the involved area. Identification of the affected areas requires instruments sensitive to geometrical properties. The SAR signal is sensitive to both the resolution and the wavelength scales; therefore, it is a good candidate for the scope.

In this paper, we presented how fractal concepts can be used both for describing the formation of the SAR signal and for extracting information from it. An overall framework was presented, providing a flexible procedure whose implementation can be tailored on several disaster monitoring cases. The combined use of direct and inverse chain gives scientists involved in the extraction of information from SAR images a powerful instrument. In particular, in this paper, main attention was focused on applications related to the monitoring of areas affected by a disaster.

The appropriate use of the framework permitted finding an empirical relation between fractal parameters of a given surface and its image. Such a result provides a great added value in the interpretation of actual SAR images. As an example, it can be possible to identify the area affected by a disaster by discriminating the fractal parameters of the postcrisis scenario, performing a SAR image classification. 
In this paper, the development of a change detection technique was provided. It is based on the use of a SAR rawsignal simulator, whose outputs were compared with actual case images with encouraging results. Then, a change detection approach for the identification of a flooded area was presented by combining the classical signal magnitude differencing technique and an innovative fractal technique, based on the differencing of the fractal parameters of the pre- and postcrisis area. The obtained results showed that the proposed combined technique leads to a significant performance improvement because it exploits the complementary information extracted by the two methods.

The presented framework is modular, and it allows quick tailoring of the algorithms to a specific situation; therefore, its use in monitoring disasters is particularly attractive. The presented studies are just examples of the potentiality of such a framework to give added value products for disaster monitoring. The tools presented in Section II can be appropriately combined in different ways according to each peculiar situation, giving the user the most adequate instrument, tailored on his needs.

\section{REFERENCES}

[1] L. Hess, J. M. Melack, S. Filoso, and Y. Wang, "Delineation of inundated area and vegetation along the Amazon floodplain with the SIR-C synthetic aperture radar," IEEE Trans. Geosci. Remote Sens., vol. 33, no. 4, pp. 896904, Jul. 1995

[2] M. Gianinetto, P. Villa, and G. Lechi, "Postflood damage evaluation using Landsat TM and ETM+ data integrated with DEM," IEEE Trans. Geosci. Remote Sens., vol. 44, no. 1, pp. 236-243, Jan. 2006.

[3] P. Lundgren, P. Berardino, M. Coltelli, G. Fornaro, R. Lanari, G. Puglisi, E. Sansosti, and M. Tesauro, "Coupled magma chamber inflation and sector collapse slip observed with synthetic radar interferometry on Mt. Etna," J. Geophys. Res., vol. 108, no. B5, pp. 2247-2262, 2003.

[4] F. Colesanti, A. Ferretti, C. Prati, and F. Rocca, "Monitoring landslides and tectonic motions with the permanent scatterers technique," Eng. Geol., vol. 68, no. 1/2, pp. 3-14, 2003.

[5] G. Franceschetti, A. Iodice, D. Riccio, G. Ruello, and R. Siviero, "SAR raw signal simulation of oil slicks in ocean environments," IEEE Trans. Geosci. Remote Sens., vol. 40, no. 9, pp. 1935-1949, Sep. 2002.

[6] P. M. Barbosa, J. M. Grégoire, and J. M. C. Pereira, "An algorithm for extracting burned areas from time series of AVHRR GAC data applied at a continental scale," Remote Sens. Environ., vol. 69, no. 3, pp. 253-263, 1999.

[7] B. B. Mandelbrot, The Fractal Geometry of Nature. New York: Freeman, 1983.

[8] K. Falconer, Fractal Geometry. Chichester, U.K.: Wiley, 1989.

[9] J. S. Feder, Fractals. New York: Plenum, 1988.

[10] R. F. Voss, "Random fractal forgeries," in Fundamental Algorithms for Computer Graphics, R. A. Earnshaw, Ed. New York: Springer-Verlag, 1985, pp. 805-835.

[11] G. Franceschetti, A. Iodice, and D. Riccio, "Fractal models for scattering from natural surfaces," in Scattering, R. Pike and P. Sabatier, Eds. London, U.K.: Academic, Sep. 2001, pp. 467-485.

[12] N. Yokoya, K. Yamamoto, and N. Funakubo, "Fractal-based analysis and interpolation of 3D natural surface shapes and their application to terrain modeling," Comput. Vis. Graph. Image Process., vol. 46, no. 3, pp. 284 302, Jun. 1989.

[13] G. Franceschetti, M. Migliaccio, D. Riccio, and G. Schirinzi, "SARAS: A SAR raw signal simulator," IEEE Trans. Geosci. Remote Sens., vol. 30, no. 1, pp. 110-123, Jan. 1992.

[14] E. Rignot and J. van Zyl, "Change detection techniques for ERS-1 SAR data," IEEE Trans. Geosci. Remote Sens., vol. 31, no. 4, pp. 896-906, Jul. 1993.

[15] M. Carlotto, "Detection and analysis of change in remotely sensed imagery with application to wide area surveillance," IEEE Trans. Image Process., vol. 6, no. 1, pp. 189-202, Jan. 1997.

[16] G. Franceschetti, M. Migliaccio, and D. Riccio, "An electromagnetic fractal-based model for the study of the fading," Radio Sci., vol. 31, no. 6, pp. 1749-1759, Nov./Dec. 1996.
[17] G. Franceschetti, A. Iodice, M. Migliaccio, and D. Riccio, "Scattering from natural rough surfaces modelled by fractional Brownian motion twodimensional processes," IEEE Trans. Antennas Propag., vol. 47, no. 9, pp. 1405-1415, Sep. 1999.

[18] M. V. Berry and Z. V. Lewis, "On the Weierstrass-Mandelbrot fractal function," in Proc. R. Soc. Lond. A, Math. Phys. Sci., Apr. 1980, vol. 370, pp. $459-484$.

[19] G. Franceschetti, A. Iodice, D. Riccio, and G. Ruello, "Extended boundary condition method for scattering and emission from natural surfaces modeled by fractals," IEEE Trans. Geosci. Remote Sens., vol. 43, no. 5, pp. 1115-1125, May 2005.

[20] M. V. Berry and T. M. Blackwell, "Diffractal echoes," J. Phys. A, Math. Gen., vol. 14, no. 11, pp. 3101-3110, Nov. 1981.

[21] G. Franceschetti, M. Migliaccio, and D. Riccio, "SAR raw signal simulation of actual scenes described in terms of sparse input data," IEEE Trans. Geosci. Remote Sens., vol. 32, no. 6, pp. 1160-1169, Nov. 1994.

[22] G. Ruello, P. Blanco, A. Iodice, J. J. Mallorqui, D. Riccio, A. Broquetas, and G. Franceschetti, "Synthesis, construction and validation of a fractal surface," IEEE Trans. Geosci. Remote Sens., vol. 44, no. 6, pp. $1403-$ 1412, Jun. 2006

[23] S. R. Brown and C. H. Scholz, "Broad band study of the topography of natural rock surfaces," J. Geophys. Res., vol. 90, no. B14, pp. 12575 12582,1985

[24] F. T. Ulaby, R. K. Moore, and A. K. Fung, Microwave Remote Sensing: Active and Passive. Reading, MA: Addison-Wesley, 1982.

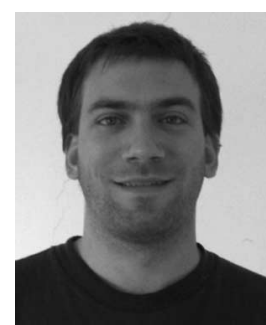

Gerardo Di Martino (S'06) was born in Naples, Italy, on June 22, 1979. He received the Laurea degree (cum laude) in telecommunication engineering from the University of Naples "Federico II," Naples, in 2005 , where he is currently working toward the Ph.D. degree.

$\mathrm{He}$ is currently with the Department of Electronic and Telecommunication Engineering, University of Naples "Federico II." His main research interests are in the field of microwave remote sensing and simulation of SAR signals, as well as the application of fractal geometry to electromagnetic scattering.

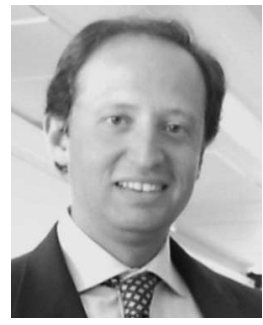

Antonio Iodice (S'97-M'99-SM'04) was born in Naples, Italy, on July 4,1968 . He received the Laurea degree (cum laude) in electronic engineering and the $\mathrm{Ph} . \mathrm{D}$. degree in electronic engineering and computer science both from the University of Naples "Federico II," Naples, in 1993 and 1999, respectively.

In 1995, he received a grant from the Italian National Research Council (CNR) to be spent at the Istituto di Ricerca per l'Elettromagnetismo e i Componenti Elettronici (IRECE), Naples, for research in the field of remote sensing. He was with the Telespazio S.p.A., Rome, Italy, from 1999 to 2000. Since 2000, he has been with the Department of Electronic and Telecommunication Engineering, University of Naples "Federico II," where he is currently a Professor of electromagnetics. He is the author or coauthor of more than 100 papers published in refereed journals or in proceedings of international and national conferences. His main research interests are in the field of microwave remote sensing and electromagnetics: modeling of electromagnetic scattering from natural surfaces and urban areas, simulation and processing of SAR signals, and SAR interferometry. 


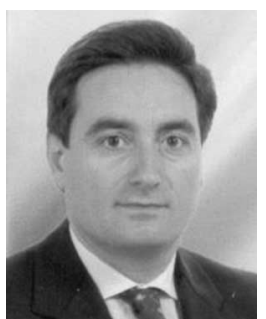

Daniele Riccio (M'91-SM'99) was born in Naples, Italy, on April 13, 1962. He received the Laurea degree (cum laude) in electronic engineering from the University of Naples "Federico II," Naples, in 1989.

$\mathrm{He}$ is a Professor of electromagnetics and remote sensing with the University of Naples "Federico II." He was a Research Scientist with the Istituto di Ricerca per l'Elettromagnetismo e i Componenti Elettronici (IRECE) of the Italian National Research Council (CNR) and with the Department of Electronic and Telecommunication Engineering, University of Naples "Federico II," Italy. In 1994 and 1995, he was a Guest Scientist with the DLR HighFrequency Institute, Munich, Germany, and in 2006, he was a Visiting Professor with the UPC, Barcelona, Spain. His research activity is witnessed by more than 150 papers published mainly in the fields of microwave remote sensing, SAR with emphasis on sensor simulation modeling and information retrieval for land oceanic, and urban scenes, as well as in the application of fractal geometry to remote sensing and electromagnetic scattering from natural surfaces.

Prof. Riccio has won several fellowships from private and public companies (SIP, Selenia, CNR, CORISTA, CRATI) for research in the remotesensing field.

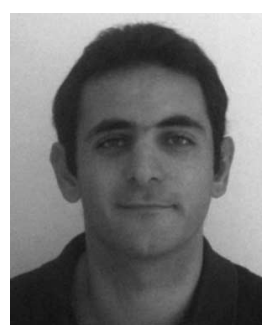

Giuseppe Ruello (S'00-M'03) was born in Naples, Italy, on February 12, 1975. He received the Laurea degree (with honors) in telecommunication engineering and the Ph.D. degree in information engineering from the University of Naples "Federico II," Naples, in 1999 and 2003, respectively.

In 2000 , he received a grant from the University of Naples to be spent at the Department of Electronic and Telecommunication Engineering for research in the field of remote sensing. In 2000, he received also a grant from University of Rome "La Sapienza," Rome, Italy. In 2002 as well as in 2004-2005, he was a Visiting Scientist with the Department of Signal Theory and Communications, Universitat Politecnica de Catalunya, Barcelona, Spain. He is currently a Research Scientist with the Department of Electronic and Telecommunication Engineering, University of Naples. His main research interests are in the field of SAR remote sensing, modeling of electromagnetic scattering from natural surfaces, SAR raw-signal simulation, modeling of electromagnetic field propagation in urban environment, and remote-sensing techniques for developing countries. 